Reporting Date: Feb. 1974

Issued: June 1974

\title{
FITWEIB: A Program to Fit Faiiure Data to Weibull Probability Parameters
}

by

G. P. Boicourt 
This report was prepared as an account of work sponsored by the United States Government. Neither the United States nor the United Stales Atomic Energy Commission, nor any of their employees, nor any of their contractors, subcontractors, or their employees, makes any warranty, express or implied, or assumes any legal liability or responsibility for the accuracy, completeness or usefulness of any information, apparatus, product or process disclosed, or represents that its use would not infringe privately owned rights.

Printed in the United States of America. Available from

National Technical Inforrnation Service

U.S. Department of Commerce

5285 Port Royal Road

Springtield, Virginiar 22151

Price: Printed Copy $\$ 4.00$ Microfiche $\$ 1.45$ 


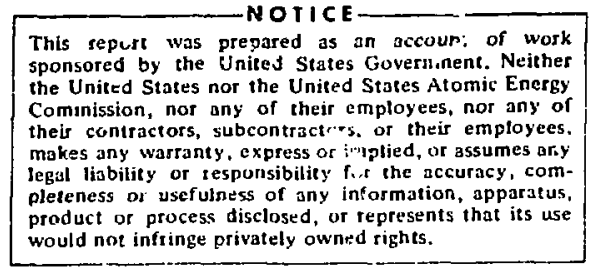

\title{
FITWEIB: A PROGRAM TO FIT FAILURE DATA TO WEIBULL PROBABILITY PARAMETERS
}

by

\section{G. P. Boicourt}

\begin{abstract}
The Weibull probability distribution describes the fuilures of many electrical and mechanical components. Tests can be carried out to obiain raw data on failures experienced by a given component type. The computer program described in this report can be used to find the parcmeters of the Weibull distribution that best fits these data. The fiting methods a:e discussed, the program is described, input instructions are given, and an example is presented.
\end{abstract}

\section{INTRODUCTION}

A prime objective of component testing is to determine whether a given component will perform satisfactorily when used as part of some larger piece of equipment. To use the failure data obtained from the tests to predict the performance of the larger system, it is advantageous to describe the failures b? a probability distribution. The Weibull ${ }^{1-3}$ distribution has greal generality and describes many mechanical and electrical components. This report describes a computer progrum that can be used to fit failure data to a three-parameter Weibull distribution and that takes into account components on which testing was suspended before their failure. The latter feature allows periodic examination of operations data on components in silu, because a componerst may still be operating and also be weighed in the analysis.

\section{THEORETICAL PRELIMINARIES}

The Weibull distribution in conplete generality is a function of three parameters; the cumulative distribution is given by

$$
F(t)=1-e^{\frac{-(t-y)^{\beta}}{\alpha}} .
$$

where

$\alpha=$ scale parameter,

$\alpha^{i 3}=$ characteristic life,

$\beta=$ shape parameter or Weibull slope,

$\gamma=$ location parameter.

Equation (1) can be written as

$$
\ln \ln \left(\begin{array}{c}
1 \\
1-\mathrm{F}
\end{array}\right)=\ln \alpha+\beta \ln (\mathrm{t}-\gamma) .
$$

which is a linear relation between the variables,

$$
y=\ln \ln \left(\begin{array}{c}
1 \\
i-F
\end{array}\right)
$$

and

$$
x=\ln (t-\gamma)
$$

The data available for fitting the distribution are the time $t_{1}$ of the $i^{\text {th }}$ failure and the fraction of the test sample which has failed through time $t_{1}$. The value of 
$t_{i}$ can be used directly in Eq. (2), but the failed fraction of the sample does not accurately reflect the fraction of the population which would have failed, especially if the sample is small. One way of estimating the ronk to be associated with a given fraction of sample failed is through use of median rank statistics. ${ }^{4}$ The procedure assigns a rank, a value of $F$ which will be too high half the time and too low half the time; hence, it is a "median" rank. Median rank tables 5 are available for samples of as many as 50 items. Johnson ${ }^{4}$ gives a formula that is good when the sample size $\mathrm{n}$ is greater than 20 :

$$
\begin{aligned}
r_{n}(j)= & \frac{j-(1-\ln 2)-(2 \ln 2-1)(j-1 / n-1)}{n} . \\
& j-1, \ldots, n .
\end{aligned}
$$

The rank assigned to the $\mathrm{j}^{\text {th }}$ failure in a sample of size $n$ is $r_{n}(j)$, provided there are no suspended items whose life is lower than $t_{j}$. Corrections for suspended items can be made by use of mean-order numbers. ${ }^{3}$ If no suspensions have occurred before the $j^{\text {th }}$ failure, the order number assigned to the $j^{\text {th }}$ failure is $j$. Let $O_{j}$ equal the order number of the $i^{\text {th }}$ failure and $m$ be the number of items still good for all $t<t_{j}$. Then,

$$
o_{j}=o_{j-1}+\frac{(n+1)-o_{j-1}}{1+m} \text {. }
$$

Note that $m$ includes the failure at $t_{i}$; that is, the inequality $t<t$ is strict. Interpolation can be used in conjunction with $\mathrm{Eq}_{\mathrm{q}}$. (3) is assign median ranks to failures with fractional order numbers.

Given values of $t_{j}$ and $F_{i}$, we now must find values of $\alpha, \beta$, and $\gamma$ which best fit them to Eq. (1), or equivalently, Eq. (2). We will work with Eq. (2) because if the value of $\gamma$ is known, values of $\ln a$ and $\beta$ can be obiained directly from a linear least-squares fit The difficulty is that we do not know $\gamma$. The literature is mostly silent about the problem of non. zero $\gamma$. There seems to be no anlaytical method of determining $\gamma$, and trial and error using the eye to determine the best value is recommended. FITWEIB uses a similar method. Least-squares fits are determined for three selected trial $\gamma$ values, and the deviations of the fits are used as criteria to change the trial values. The value of $\gamma$ obtained is that which yields the smallest deviation.

A word of caution is necessary here. FIT'WEIB assumes that $\gamma$ is not zero and that only a single distribution is present and tries to fnd a siraight line fit for Eq. (2). Components in a given sample often fail by two or more different mechanisms. These failures can lead to data that appear as though a single mechanisn were operative, but with a nonzero $\gamma$. If enough data are available, it is usually easy to distinguish betweess the two cases. When data are sparce and scattared, it is not easy. FITWEIb plots the raw data to enable the user to check the plot.

FITWEIB fits this data for two sets of weights. One fit is made using a weight of $l$, that is no weighting. A second fit is made using weights proportional to the reciprocal of the proint's variance, assuming that the variance of $F$ aboust its expected value is the same for all values of $F$. Thus, for the case at hand,

$$
w_{i} \propto \frac{1}{\sigma_{i}^{2}}
$$

$$
\sigma_{i}^{2} \propto\left\{\frac{d}{d F}\left[\ln \ln \left(\frac{1}{1-F}\right)\right]\right\}^{2}
$$

and

$$
\propto \frac{1}{\left[\ln \left(\frac{1}{1-F}\right)\right]^{2}} \frac{1}{\left(1-i^{2}\right)^{2}}
$$

This gives

$$
W_{i}=(1-F)^{2}\left[\ln \left(\frac{1}{i-F}\right)\right]^{2}
$$

Equation (5) is the theoretically correct weight, assuming uniform variance of $F,{ }^{6}$ and it should give the preferred fit. However, the unweighted fit is in. cluded because it tends to emphasize the early failures and could be valuable in distinguishing whether two friture distributions are present.

There is a second reason for providing two fits. When the data include a large number of items on which tests were suspended belore icilure of the last failed item, convergence may not occur, because of inaccuracies in the data. In these circumstances, the fit with a weight ol 1 will often converge when the fit with the correct weight will not.

\section{DESCRIPTION OF THE PROGRAII}

Figure $l$ is a simplified flow diagram of the program, a listing is given in Appendix $A$ and an example is provided in Appendix B. The somple size 


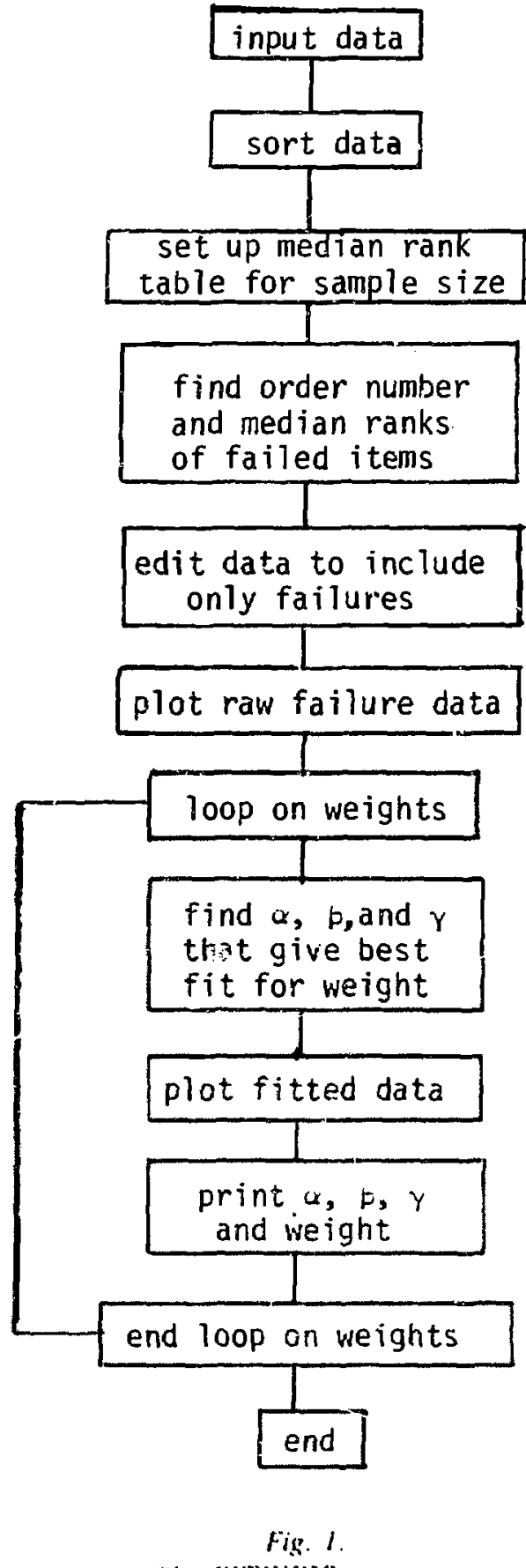

The FTTWLIB program.

and the necessaty data are read and sorled to place the lives in ascending order. AANK is called and sets up a median rank table for a sample of the given size. Then a loop on the items assigns an order number and median rank to each tailed item. The data are then edited to eliminate the suspended items that are no longer needed. The raw failure data can be plotted at this point. Next, the failure data are fitted to find the best $\alpha$, $\beta$, and $\gamma$ values, the fitted data are plotted, and the values of $\alpha, \beta$, and $\gamma$ are printed; this is done for each weight.

\section{FUNCTIONS OF FITWEIB SUEROUTINE:}

Subroutine

Function

\section{FITWEIB Driver for program.}

1. INDATA Reads sumple size (N), number of items being read in (NF), and the code number for the time unit desired on plots (IUNIT). IUNIT may have the following values

$\begin{array}{cl}\text { IUNIT } & \text { TIME UNIT } \\ 1 & \text { shois } \\ 2 & \text { seconds } \\ 3 & \text { hours } \\ 4 & \text { days } \\ 5 & \text { operations }\end{array}$

NF test items (LIFE(I)) are read along with a failure code (IFL(I)), 1 if a failure, otherwise 0 to indicate whether the unit failed or was suspended. The input data are printed out.

2. SORT The life dala are put in ascen. ding order, and NF is set to the index of the longest lived failure.

3. RANKS A median rank table is com. puted for sample size $\mathrm{N}$. If $N \leq 20$, ranks are taken from $a$ table, if $\mathrm{N}>20$, they are computed using Eq. (3).

4. ORDRNK An order number and median rank are assigned to each failed item.

5. AANCO Called by ORDRNK. Calculates the median rank for an item having eil'.er integer or noninteger order number. 
6. EDIT Edits the vector LIFE. After the call to EDIT, LIFE holds only failures and NF corresponds to the actual number of failures.

7. WBLPRAM Calculates the Weibull parameters $\alpha, \beta$, and $\gamma$ using an interative search. Three values of $\gamma$ are assumed, fits to obtain $\alpha$ and $\beta$ are made for each value of $\gamma$, and the deviations of the fits are used to improve the $\gamma$ values.

8. DEVIA Called by WBLPRAM. Makes three weighted least-squares fits for the three proposed $\gamma$ values.

9. PLOTWIB Driver subroutine for producing Weibull plots.

10. PLOWB, Plotting routines used to WLGLNTM produce a standardized and WLNLN Weibull plot. PLOWB is based in part on the LASL library routine PLOJB; WLGLNTM, in part on two LASL library routines, DLNLGTM and DLNLG; and WLNLN, in part on LASL library routine DLNLN. These routines call a number of other LASL systems plotting routines.

\section{ACKNOWLEDGMENT}

I would like to express my appreciation to Mrs. Verna L Gardiner for her help in rewriting the plotting routines.

\section{REFERENCES}

1. W. Weibull, "A Statistical Distribution Function of Wide Applicability," ]. Appl. Phys. 18, 293-297 (1951).

2. J. H. K. Kao, "A New Life-Quality Measure for Elecíron Tubes," IRE Tranis. Reliability Quality Control (IEEE PGRQC) 7, 1-11 (April 1956).

3. L. G. Johnson, The Statistical Treatment of Faligue Experiments (Elsevier, Amsterdam, 1964).

4. L. G. Johnson, "The Median Ranks of Sample Values in the Population with an Application to Certain Fatigue Studies," Ind. Math. 2, 1.9 (1951).

5. L. G. Iohnson, Theory and Technique of Variation Research (Elsevier, Amsterdam, 1964).

6. C. Daniel and F. S. Wood, Fitring Equations to Data (Wiley-Interscience, New York, 1971).

\section{INPUT REOUIREMENTS}

Title card (6A10)

Cols $\quad 1-60$

Control Card (2I6)

1.6 Number of items in test sample $\mathrm{N}$

7.12 Number of items to be read in NF

LIFE DATA CARDS (F12.3, I12) NF in number

1.12 Life LIFE (I)

13-24 Failure code IFL (I) 


\section{APPENDIX A}

\section{PROGRAM LISTING OF FITWEIB}

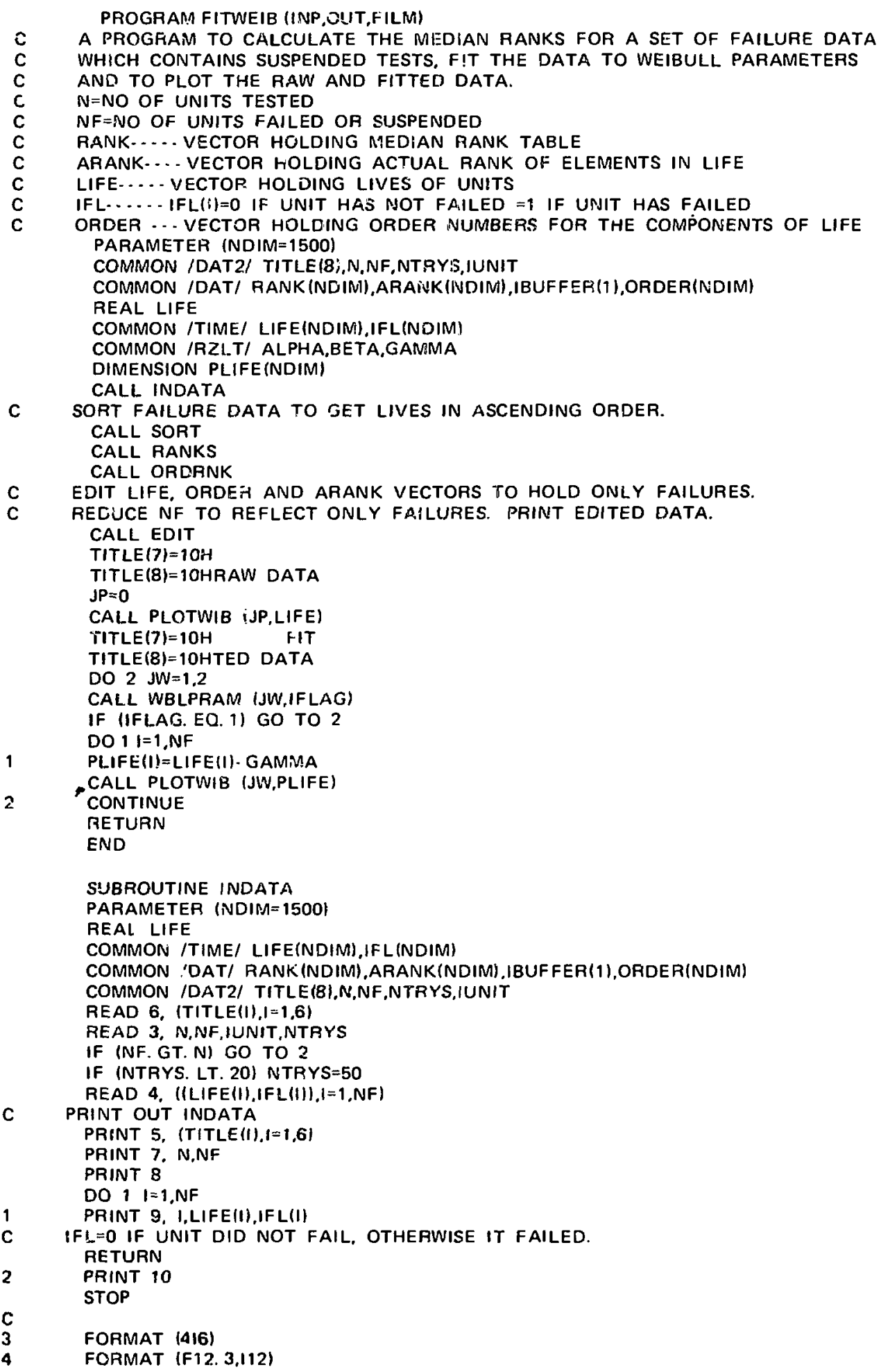

FITW0001

FITW0002

FITW0003

FITW0DO4

-ITWOLO5

FITW0006

FITW0007

FITWOOOB

FITW0009

FITWN010

FITW0011

FITW0012

FITVU0013

FITW0014

FITW0015

FITW0016

FITW0017

FITW0018

FITW0019

FITW0C20

FITWOC21

FITW0022

FITW0023

FITW0024

FITW0625

FITW0026

FITW0027

FITWC028

FITW0029

FITW0030

FITW0031

FITW0032

FITW0033

FITW0034

FITW0035

FITW0036

FI'TW0037

FITWOC38

FITWUO3S

FITW0040

FITW0041

INDATOO

INDATOO:

INDAT003

INDAT004

INUAT005

INDAT006

INDAT007

INDAT008

INDATOO9

INDAT010

INDAT01!

INDAT012

INDAT013

INDAT014

INDAT015

INDAT016

INDAT017

INDAT018

INDAT019

INDAT020

INDAT021

INDAT022

INDA.T023

INDAT024 
SUBROUTINE SORT

SORTOOO1

PARAMETER (NDIM) 1500 )

COMMON /TIME/ LIFE(NDIM),IFL(NDIM)

REAL LIFE

COMMON /DAT2/ TITLE(8),N,NF,NTRYS,IUNIT

$N F M 1=N F-1$

D) $2 \quad I=1$, NFM1

$\mid P 1=1+1$

DO $1 J=\mid P 1$,NF

IF (LIFE(!). LE. LIFE(J)) GO TO 1

TEMP=LIFE(I)

LIFE(I)=LIFE (J)

LIFE $(J)=$ TEMP

ITEMP=IFL(I)

IFL( ()$=\mid F L(J)$

! $F L(J)=$ ITEMP

CONTINUE

CONTINUE

ADJUST NF IF HIGHEST LIFE IS NOT A FAILURE.

DO $3 \quad I=1$,NF

$N F=N F-1+1$

RETURN

END

STRTOOO2

SCRTO003

SORT0007

SORT0005

SORT0006

SORTO007

SOST0008

SORT0009

SORTO010

SORTO01 i

SORTO012

SORT0013

SORTO014

SORT0015

SORT0016

SORT0017

SORT0018

SORT0019

SORTUU20

SORTO021

SORTO022

SORT0023

SORTO024

SUBROUTINE RANKS

C ROUTINE COMPUTES A MEDIAIY RANK TABLE FOR A TEST SAMPLE OF SIZE $N$ PARAMETER (NDIM $=1500$ )

COMMON /DAT/ RANK(NDIM),ARANK(NDIM),IBUFFER(1),ORDER(NDIM)

COMMON /DAT2/ TITLE(8),N,NF,NTRYS,IUNIT

DIMENSION R(210)

DATA (R(1), $\mid=1,171) / .5, .29289, .70711$.. 2063,. 5.. 7937.. 1591,. 38573,

: 61427,.8409,. 12945,. 31381,. 5,.68619,.87055,. 1091,. 26445,. 42141,. 57

$2859, .73555, .8909,09428, .22849, .36412, .5 . .63588, .77151, .90572,083$

3 .. 20113.. 32052,. 44015,. 55984,. 67948,. 79887,. 917,. 07412,. 17962,. 286

$424, .39308, .5,60691, .71376, .82038, .92587, .06697, .16226, .25857, .355$

$5 \quad 1.45169 .54831,6449.74142, .83774,93303.06107, .14796, .23578, .3$

$6238, .41189, .5, .58811, .6762, .76421_{f} .85204, .93893, .05613, .13598,216$

7 69. 29758,. 37853,. 45951,. 54049,.62147,. 70242,. 78331,. 86402,. 94387,

8 .05192,. 12579.. 20045,. 27528,. 35016.. 42508,. 5, 57492,.64984,. 72472.

$9.79955, .87421, .94808, .0483, .11702, .1864 \%, 25608, .32575,39544, .465$

$\$ 15, .53485, .60456, .67425, .74392,81353, .88298, .95169,04516, .1094$.

$\$ 17432, .23939 .30452$. 36967.. 43483. 5.. 56517.63033..69548,. 76061,.

$\$ 82568,8966, .95484, .0442, .1027, .16365, .22474, .28589$. 34705,. 40823,

$\$ .46941,53059.59177,65295, .71411,77525,83635, .8973 .9576,0399$

$\$ 5, .09678, .15422, .21178 . .2694, .32704, .38469, .44234, .5, .55766, .61531$

$\$ \quad 67296,7306,78821,84578,90322,96005,03778,09151,14581,20$

$\$ \quad 024.25471, .30921,36371_{, .} 41223.47274, .52726,58177, .63629, .69079$

$\$$. 74529, 7S976,. 85419,. 90849,.96222

DATA (R(I), $\mathrm{f}=172,210) \%$ 03582,.08677,. 13827,. 18988, 24154, 29322,. 3

$14491, .3966, .4483, .5, .5517, .6034, .65509, .70678, .75846,81011,86173$

$2.91322,96418,03406,08251,13147,18055,22967, .2788,32795 \ldots 37$

$371 . .42626 . .47542, .52458, .57374, .62289 .67205 .72119, .77033 .81945$,

$4.86853, .91749, .96594 /$

IF (N. LE. 20) GO TO 2

$Q=1$. - ALOG(2.)

$S=2$. $A L O G(2)-$,1 .

RANKSED1

RANKSO02

RANKS003

RANKSO04

RANKSOO5

RANKSOO6

RANKSCO7

RANKS008

RANKSOO9

RANKS010

RANKSO11

RANKS012

RANKSO13

RANKS014

RANKS015

RANKS016

RANKS017

RANKS018

RANKS019

FANKSO2O

RANKSO? 1

RANKSO22

RANKS023

RANKS024

RANKSO25

RANKS026

RANKSO27

RANKSO28

RANKSO29

RARK S030

RANKSO31

RANKS032 
SUBROUTINE RANCO(I)

C A SUBRGIUTINE TO CALCULATF THE MEDIAN RANK FOR A DATA POINT

RANCO001 C HAVING INTEGER OR NONINTEGER ORDER NUMBER

C I INDEX OF ORDER MUMBER

C $K$ LARGEST INTEGER EQUAL TO OR LESS THAN ORDER(i) PARAMETER (NDIM=1500) COMMON /DAT/ RANK(NDIM),ARANK(NDIM),IBUFFER(1),ORDER(NDIM! $K=$ INT (ORDER(I))

DO $2 \mathrm{~J}=\mathrm{K}, \mathrm{N}$

IF (ORDER(I). GT. FLOAT(J)) GO TO 2

IF (ORDER(I). EQ. FLCAT(J)) GO TO 1

ARANK $(1)=\operatorname{RANK}(J-1)+(\operatorname{RANK}(\mathrm{J}) \operatorname{RANK}(\mathrm{J}-1)) *(\operatorname{ORDER}(1)-$ FLOAT(J-1)) GO TO 3

$1 \quad$ ARANK $(3)=$ RANK $(J)$

GO TO 3

2 CONTINUE

3 RETURN

END

RANCO002

RANCO003

RANCOOCA

RANCO005

RANCO006

RANCO007

RANCODOB

RANCOOO9

RANCO010

RANCO011

RANCO012

RANCO013

RANC0014

RANCO015

RANCO016

RANCO017

RANCO018

SUBROUTINE ORDRNK

PARAMETER (ND!M=1500)

COMMON /DAT2/ TITLE(8),N,NF,NTRYS,IUNIT

COMMGN /DAT/ R.NK(NDIM),ARANK(NDIM),IBUFFER(1),ORDER(NDIM)

REAL LIFE

COMMON /TIME/ LIFE(NDIM),IFL(NDIM)

DORDER $=1$.

$\operatorname{ORDER}(0)=0$

DO $2 \quad i=1$, NF

$j=0$

IF (IFL(I). EO. O) GO TO 1

ORDER $(1)=$ DORDER+ ORDER(1-1)

CALL RANCO (I)

GO TO 2

1

$\mathrm{J}=\mathrm{J}+1$

IF (IFL(1+J). EO. O) GO TO I

DORDER $=(F L O A T(N+1) \cdot$ ORDER(I-1))/1 + + FLOAT(N- $(1+J-1))$

$\operatorname{ORDER}(1+\mathrm{J})=$ DORDER+ $\operatorname{ORDER}(\mid-1)$

$I=I+J$

CALL RANCO (I)

CONTINUE

C

RETURN

END

ORDROOO

ORDROCO2

OROROOO3

ORDRUOO4

ORDROOG5

ORDROOO6

ORDFOO07

ORDROODR

ORDROOU9

ORDR0010

ORDR0011

ORDR0012

ORDR0013

ORDR0014

ORDR0015

ORDR0016

ORDR0017

ORDR0018

ORDF0019

ORDF,0020

ORDR0021

ORDR0022

ORDR0023

ORDR0024

SUBROUTINE EOIT

EDITO001

PARAMETER (NDIM $=1500)$

EDITON02

COMMON IDAT2/ TITLE(8),N,NF,NTRYS,IUNIT

COMMON /DAT/ RANK(NDIM),ARANK(NDIM),IBUFFER(1),ORDER(NDIM)

EDIT0003

REAL LIFE

COMMON / TIME/ L.IFE(NDIM),IFLINDIM)

INF $=0$

DO $1 \quad 1=1$,NF

IF (IFL(I). EO. O) GO TO 1

EDITOCO4

EDIT0005

EDIT0006

EDITO007

EDITOOOB

EDITO009

INF $=I N F+1$

EDIT0010 


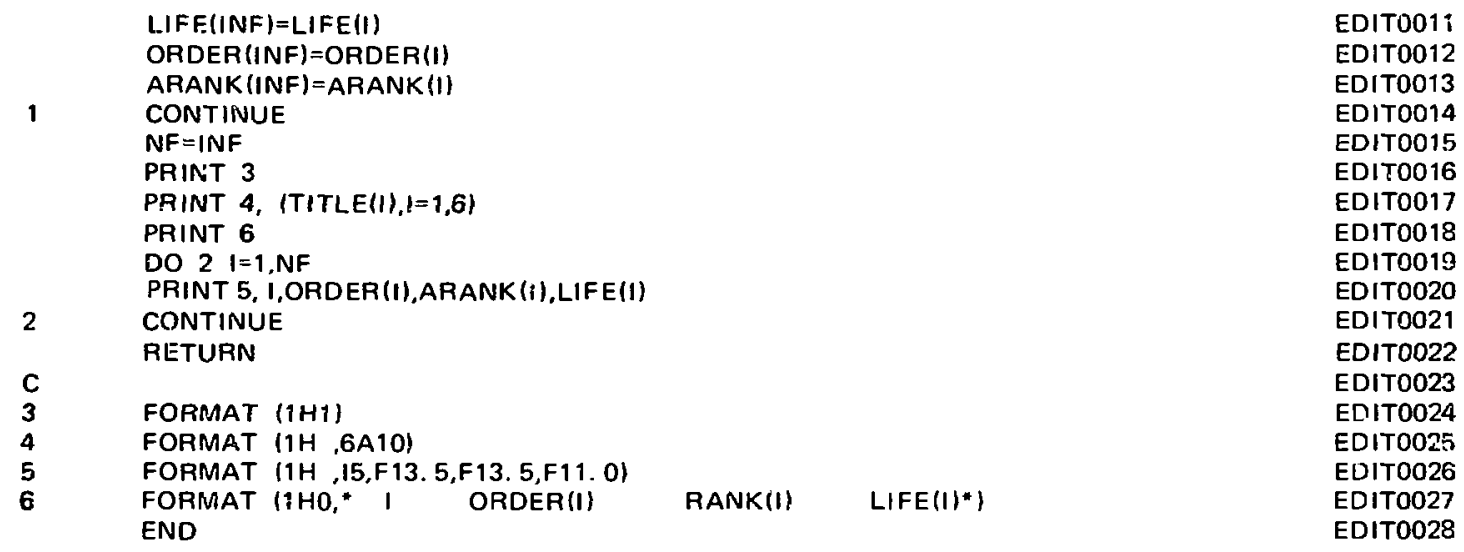

SUBROUTINE WBLPRAM(IWEIGHT,IFLAG)

PARAMETER (NDIM $=1500$ )

COMMON /DAT/ RANK(NDIM),ARANK(NDIM),JBUFFER(1),ORDER(NDIM)

COMMON /DAT2/ TITLE(8),N,NF,NTRYS,IUNIT

COMMON /RZLT/ ALPHA,BETA,GAMMA

DIMENSIONN WEIGHT(2)

REF.L LIFE

COMMON /TIME/ LIFE(NDIM),IFLINDIM!

. COMMON /DEVI/ X(NDIM),Y(NDIM),POS(3),DEV(3),AO(3),A1(3),W(NDIM)

C ROUTINE FINDS THE ALPHA, BETA AND GAMMA PARAMETERS OF A WEIBULL
C DISTRIBUTION SATISFIED BY THE FAILURE DATA IN LIFE. DATA IS

C ASSUMED ORDEFED. MEDIAN RANKS ARE IN ARANK.

DATA WEIGHT/1OH1

10H1/VARIANCE/

GO TO (1,3), IWEIGHT

DO $2 \quad I=1$, NF

$Y(I)=A L O G(A L O G(1 . / 11$. - ARANK(IIH)

2

$W(1)=1.0$

GO TO 5

3

DO $4 \quad I=1$, NF

TEMP = ALOG 1 1. $/ 1$. - ARANK $(1) \|$

$Y(1)=A I$.OG $(T E M P)$

$4 \quad W(1)=\left(\right.$ TEMP $\left.^{*} * 2\right) *(11$. ARANK $\left.(1)) * 2\right)$

5 CONTINUE

$\operatorname{POS}(1)=0$

NFM2 $=N F \cdot 2$

DO $6 \quad 1=1$.NFM2

6 IF (LIFE(I). NE. 0.) GO TO 7

$7 \quad \operatorname{POS}(2)=-$ LIFE (1)

$D E L=P O S(2)$

POS $(3)=P O S(2)+D E L$

DO $13 \mathrm{~J}=1$,NTRYS

FALL DEVIA (IFLAG)

IF (IFLAG. EQ. 1) RETURN

IF (DEV(1). GT. DEV(2). A. DEV(2). GT. DEV(3)) GO TO :

IF (DEV(1). LT. DEV(2). A. DEV(2). LT. DEV(3)) GO TO 11

IF (DEV(1). EC. DEV(2). A. DEV(2). L.T. DEV(3)) GO TO 9

IF (DEV(1). GT. DEV(2). A. DEV(2). LT. DEV(3)) GO TO 10

C

MINIMUM IS BETWEEN POS(2) AND POS(3)

IF (ABS(POS(2)-POS(3)). LT. . 1) GO TO 15

$\operatorname{POS}(1)=\operatorname{POS}(2)$

$\operatorname{POS}(2)=.5^{*}(\operatorname{POS}(1)+\operatorname{POS}(3))$

$D E L=.5^{\circ} \mathrm{DEL}$

GO TO 13

C MINIMUM NOT YET REACHED CONTINUE

$\operatorname{POS}(1)=\operatorname{POS}(2)$

$\operatorname{POS}(2)=\operatorname{POS}(3)$

$\operatorname{POS}(3)=\operatorname{POS}(3)+D E L$

GO TO 13

WBLPR00 1

WBLPRO02

WBLPR003

WBLPR004

WBLPR005

WBLPRO06

WBLPR007

WBLPRO08

WBLPR009

WBLPR010

WBLPR011

WBLPR012

WBLPR013

WBLPR014

WBLPR015

WBLPR016

WVBLPR017

WBLPR018

WBLPR019

WBLPR020

WBLPR021

WBLPR022

WBLPR023

WBLPR024

WBLPR025

WBLPR026

WBLPR027

WBLPR028

WBLPR029

WBLPR030

WBLPR031

WBLPR032

WBLPR033

WBLPR034

WBLPR035

WBLPR036

WBLPR037

WBLPR038

WBLPR039

WBLPR040

WBLPRO4 1

WBLPR042

WBLPR043

WBLPR044

WBLPR045

WBLPR046

WBLPR047

WBLPRO4R 
$D E L=.5^{*} D E L$

GO TO 13

C MININIUM BETNEEN POS(1) AND POS(3)

10 If (ABS(POS(1)-POS(2)). LT. . 1) GO TO 15

$D E L=.4 * D E L$

$\operatorname{POS}(2)=\operatorname{POS}(1)+\mathrm{DEL}$

$\operatorname{POS}(3)=\operatorname{POS}(2)+D E L$

GO TO 13

C MINIMUM IS EITHER LESS THAN POS(1) OR BETWEEN POS(1) AND POS(2)

11 IF (J. EO. 1) GO TO 12

$D E L=.5 * D E L$

$\operatorname{POS}(3)=\operatorname{POS}(2)$

$\operatorname{POS}(2)=\operatorname{POS}(1)$

POS(1)=POS(1). DEL

Go TO 13

C MINIMUM IS GREATER THAN ZERO.

12 DEL=- DEL

$\operatorname{POS}(2)=D E L$

$\operatorname{POS}(3)=\operatorname{POS}(2)+D E L$

13 CONTINUE

DO 14 NT $=1,5$

14 PRINT 16, NTRYS,WEIGHT(IWEIGHT)

$15 \quad A L P H A=E X P(-A O(2))$

PRINT 17, ALPHA,A1(2),POS(2),WEIGHT(IWEIGHT)

GAMMA=POS(2)

$B E T A=A 1(2)$

RETJRN

C

FORMAT $11 \mathrm{H}$, " ITERATION DID NOT CONVERGE AFTER*.13," PASSES WITH W

1 EIGHT*.ATOI

17

FORMAT $11 \mathrm{H}, /^{*}$ ALPHA=*,E13. 7,* BETA=*,E13. 7,* GAMMA=*,E13. 7,* WEIG

$1 H T=*, A 10)$

END

WBLPR050

WBLPR051

WBLPR052

WBLPR053

WBLPR054

WBLPR055

WBLPR056

WBLPR057

WBLPH058

WBLPR059

WBLPR060

WBLPR061

WBLPR062

WBLPR063

WBI.PR064

WBLPRO65

WBLPR066

WBLPR067

WBLPRO68

WBLPR069

WBLPR070

WBLPR071

WBLPR072

WBLPR073

WBLPR074

WBLPR075

WBLPR076

WBLPR077

WBLPR078

WBLPR079

WBLPROBO

WBLPR081

WBLPR032

WBLPR083

WBLPRO84

VJBLPR085

SUERDUTINE DEVIA(IFLAG)

DEVIA001

PARAMETER (NDIM) $=1500)$

DEVIA002

COMMON /DAT/ RANK(NDIM),ARANK(NDIM),IBUFFER(1),ORDER(NDIM)

DEVIA003

COMMON /DAT2/ TITLE(8),N,NF,NTRYS,IUNIT

REAL LIFE

COMMON /TIME LIFE(NDIM),IFLINDIM)

COMMON /DEVI/ X(NDIM), Y(NDIM),POS(3),DEV(3),AO(3),A1(3),W(NDIM) IFLAG $=0$

C FIND STARTING INOEX

IF (AMAX1(POS(1),POS(2),POS(3)). LT. LIFE(1)) GO TO 3

NFM $1=N F \cdot 1$

DO $1 \quad I=1$,NFM1

1

IF (POS(3). LT. LIFE(I)) GO TO 2

IFLAG =1

PRINT 8

GO TO 7

$2 \quad N I=1$

GO TO 4

$\begin{array}{ll}3 & N I=1 \\ 4 & \text { DO } 6 J=1,3\end{array}$

SWEIGHT $=0$.

WXSUM=0.

WXSOSUM $=0$.

WXYSUM $=0$.

WYSUM $=0$.

WYSOSUM $=0$.

DO $5 \quad I=N I, N F$

$A L=L I F E(I)-P O S(J)$

$X(1)=A L O G(A L)$

SWEIGHT $=$ SWEIGHT $+W(1)$

DEVIAO04

DEVIA005

DEVIAOO6

DEVIA007

DEVIAO08

DEVIA009

DEVIA010

DEVIA011

DEVIA012

DEVIA013

DEVIAO14

DEVIA015

DEVIA016

DEVIA017

DEVIA018

DEVIA019

DEVIA020

DEVIA021

DEVIA022

DEVIA023

DEVIAO24

DEVIA025

DEVIA026

DEVIA027

DEVIA028

DEVIA029

DEVIA030

$W \times S U M=W \times S U M+X(I) * W(I)$

DEVIA031 
WXSOSUM $=W X S Q S U M+X(1) * 2 * W(1)$

DEVIA032

$W X Y S U M=W X Y S U M+Y(1) * X(1) * W(1)$

DEVIA033

WYSUM=WYSUM+ $Y(1) * W(i)$

$W Y S O S U M=W Y S O S U M+Y(1) * * 2 * W(1)$

DEVIA034

CONTINUE

FIND A1 AND AO

=1. /(SWEIGHT*WXSOSUM-WXSUM**2)

DEVIA035

DEVIA036

DEVIA037

$A 1(J)=\left(S W E I G H T * W X Y S U M-W Y S U M{ }^{*} W X S U M\right) * D$

DEVIA038

$A O(J)=(W Y S U M * W X S O S U M-W X Y S U M * W X S U M) * D$

DEVIA039

DEV $(J)=W Y S O S U M-A O(J)$ *WYSUM- A1 (J) *WXYSUM

DEVIAO40

DEVIAO41

CONTINUE

RETURN

DEVIA042

DEVIA043

DEVIA044

FORMAT 11H * POSITION 3 IS . GE. 2ND GREATEST LIFE. NO FIT CAN BE

DEVIA045

1 MADE FOR THIS WEIGHT. ")

DEVIA046

END

DEVIA047

SUBROUTINE PLOTWIB(JP,LIFE)

PARAMETER (NDIM=1500)

COMMON /RZLT/ ALPHA,BETA,GAMMA

DIMENSION AFA(2), BTA(2), GA(2), WGT $(3,2)$

COMMON /DAT/ RANK(NDIM),ARANK(NDIM),IBUFFER(1),ORDER(NDIM)

COMMON /DAT2/ TITLE(8),N,NF,NTRYS,IUNIT

COMMON /RTLIM/ $\times 2$

DIMENSION LABELX(6), WEIBSCA(NDIM), LABELY(2), X(2), Y(2)

REAL LIFE(NDIM)

DATA LABELY/10HPERCENT FA,10HILED

DATA LABELX/10H SHOTS, $10 \mathrm{H}$ SECONDS, $10 \mathrm{H}$ HOURS, $10 \mathrm{H}$ DAYS

1 , 10HOPERATIONS!

11 DATA AFA(1),BTA $\{1\}, \mathrm{GA}(1) / 10 \mathrm{H}$ ALPHA $=, 10 \mathrm{H}$ BETA $=, 104$ GAMMA $=$

DATA WGT(1,1),WGT(2,1),WGT(3,1)/10H WEIGHT IS, 10H 1. 0,1H /

DATA W.ST(1,2),WGT(2,2),WGT(3,2)/10H WEIGHT IS,10H 1/VARIANC,1HE!

DO $1 \quad \mid=1$, NF

WEIBSCA $(1)=$ ALOG $(A L O G(1 . /(1,-$ RANK $(1))))$

DO $2 \quad I=1$,NF

3

IF (LIFE(I). GT. O.) GO TO 3

NS $=1$

$N F F=N F+1-N S$

IF (NS. EQ. 1 ) GO TO 5

DO $4 i=N S$ NF

$K=1+1$ - NS

LIFE(K)=LIFE(I)

4 WEIBSCA $(K)=$ WEIBSCA(1)

5 L=- NFF

CALL PLOWB (LIFE,WEIBSCA,L,46, TITLE,80, LABELX(IUNIT),10,LABELY,20)

IF (JP. EQ. O) GO TO 6

C

DRAW FIT LINE

$X(1)=0$.

$Y(1)=-A L O G(A L P H A)$

$X(2)=X 2$

$Y(2)=Y(1)+B E T A * X(2)=2.302585$

CALL PLOT $(2, X, 1, Y, 1,46,1)$

ENCODE $(10,7$,AFA(2))ALPHA

ENCODE $(10,7, B T A(2)) B E T A$

ENCODE $(10,7$, GA(2))GAMMA

CALL TSP $(120,952,20, A F A)$

CALL TSP $(120,968,20, B$ TA)

CALL TSP $(120,984,20, G A)$

CALL TSP $(120,1000,21, W G T(1, J P))$

6 RETURN

FORMAT (F 10. 4)

END

PLOTW001

PLOTW002

PLOTW003

PLOTW004

PLOTW005

PLOTWOOG

PLOTW007

PLOTWO08

PLOTWO09

PLOTW010

PLOTW011

PLOTW012

PLOTW013

PLOTW014

PLOTW015

PLOTW016

PLOTW017

PLOTW018

PLOTW019

PLOTW020

PLOTW021

PLOTW022

PLOTW023

PLOTW024

PLOTW025

PLOTWO26

PLOTW027

PLOTW028

PLOTWO29

PLOTW030

PLOTW031

PLOTW032

PLOTW033

PLOTW034

PLOTW035

PLOTW036

PLOTW037

PLOTW038

PLOTW039

FLOTWO4O

PLOTWO41

PLOTWO42

PLOTW043

PLOTWOA4

PLOTWO45

PLOTWO46

PLOTWO47 
SUBROUTINE PLOWB(X,Y,NPTS,NSYM,LABELZ,NZL,LABELX,NXL,LABELY,NYL)

LIN $=-1$

KSYM=IABS(NSYM)

KINC $=1$

MPTS $=\mid$ ABS(NPTS)

$M Z L=\mid A E S(N Z L)$

$N X N=\mid A B S(N X L)$

NYN $=$ IABS $\{N Y L\}$

CALL ADV (1)

CALL MAXV (X,KINC,MPTS,ISUB,XMX)

$Y M X=2.0$

$Y M N=-10$.

C THE LOG AXES WILL BE FULL CYCLES.

$X M N=0$.

$X M X=A L O G 10(X M X)$

IF (YMN. NE. YMX) GO TO 1

$D Y M=.001 * A B S(Y M X)$

IF (DYM. EQ. O) $D Y M=.0001$

$Y M N=Y M N-D Y M$

$Y M X=Y M X+D Y M$

$1 \quad$ CALL ASCL (5,YMN,YMX,MAJY,MINY,KKY)

$I X L=120$

IYT $=50$

IXR $=980$

IYB $=910$

CALL FRAME $(|X L| X R,,|Y T| Y B$,

CALL DGA (IXL,IXR,IYT,IYB,XMN,XMX,YMX,YMN)

CALL WLGLNTMI (MAJY)

CALL SBLOG

CALL SRLIN (MAJY,KKY)

CALL EXL

IF iNXN. NE. O) CALL DLCH (MAX0(54,1XL+(IXR-iXL-12*NXN)/2),IYB+60,NX

1 N,LABELX,1)

INCX $=34$

IF (NYN. GT. 85) INCX $=18$

!F (NYN. NE. O) CALL DLCV (INCX MINO(IYB+52,IYB- (IYB- IYT- 12*NYN)/2),

1 NYN,LABELY,1)

IF (NZL. NE. 0) CALL DLCH (MAXO(54,1XL+(|XR-IXL-12*MZL)/2),10,MZL,LA

1 BELZ,1)

CALL EXH

C

PLOT POINTS

DO 3 NXP=1,MPTS

$X T W O=X(N X P)$

$Y T W O=Y(N X P)$

$X T W O=A L O G 10(X T W O)$

CAI.L CONVRT (XTWO,NXTWO,XMN,XMX,|XL,IXRI

CALL CONVRT 'YTWO,NYTWO,YMN,YMX, IYB,IYT)

CALL EXL

DO $2 \quad I=1,4$

2 CALL PLT (NXTWO,NYTWO,42)

CALL EXH

NYONE=NYTWO

NXONE $=$ NXTWO

3 CONTINUE

RETURN

END

PLOWB042

PLOWBO45

PLOWBO4A

PLOWB045

PLOWBOA6

PLOWB047

PLOWB048

PLOWB049

PLOWB050

PLOWB051

PLOWB052

PLOWB053

PLOWB054

PLOWB055

PLOWB056

PLOWB057

PLOWB058

SUBROUTINE WLGLNTM(NX)

WLGLNOO1

COMMON /CJEO7/ IXL,IXR,IYT,IYB,XL,XR,YT,YB

WLGLNOO2

DIMENSION XY(4), IXY(4)

EQUIVALENCE $(X Y, X L),(I X Y, I X L)$

DIMENSION ALG(B)

COMMON /RTLIM/ $\times 2$

DATA (ALG(K),K=1,8)/. 301029995663981,. 477121254719662,. 60205999132

1 7962.. 698970004336019.. 778151250383644,. 845098040014257.. 903089986

2 991944,. 954242509439325/

DIMENSION HEAD1(2), HEAD2(2)

WLGLNOO3

WLGLNO04

WLGLNOO5

WLGLNOO6

WLGLNO07

WLGLN008

WLGLN009

WLGLNO10 
DATA HF.AD1/BH XL=XR. .8H YT=YB. I

DATA HIEAD2/9H NO OF $X, 9 H$ NO OF $Y I$

DATA ITIC/10/,ITICI/15/

CALL WLNLNTM $(0, N X)$

CALL GYA (IYT,IYB,IXR)

ITYPE=I

IEX $=1$

11=3०ITYPE- 2

$12=1$ TYPE+ 1

$X 1=X Y(11)$

$X 2=X Y(12)$

IF (X1.NE. X2) GO TO I

PRINT 13, HEADIIITYPE)

$\times 2=\times 2+.01$

$X$ MIN $=$ AMIN $1(X 1, \times 2)$

$X M A X=A M A X 1(X 1, \times 2)$

XMIN=AMIN1 (AINT (XMIN), SIGNIAINT(ABS(XMIN)+ , 999), XMIN))

XMAX =AMAXIIAINT(XMAX),SIGN(AINT(ABS(XMAX) + , 999), XMAX))

$X !=X M I N$

$\times 2=X M A X$

$N Y=A B S(X 1-X 2)$

IF (NY. LE. 25) GO TO 2

PAINT 14, HEAD2(ITYPE)

RETURN

IF (NY. NE. O) GO TO 3

$Y T T=X 1+1$.

IF $(\times 2$. LT. X1) YT $>\times 1.1$.

NY $=1$

$X 1=Y T$

3 IF (X'Y(12). GE. $X Y(11)) 4,5$

IREV $=1$

$X Y(11)=X 1$

$X Y\{12\}=X 2$

GO TO 6

5 IREV $=2$

$X Y(11)=X 2$

$X Y(12)=X 1$

$6 \quad$ ISL $=(|X Y(12) \cdot| X Y(11)) / N Y$

IYC $=$ IXY(II)

DO 11 I01,PY

DO $10 K=1,8$

GO TO $(7,8)$, IREV

$7 \quad$ ICY $=A L G(K) \cdot I S L+I Y C$

GO TO 9

$8 \quad$ ICYa(1. - ALG(K))・ISL+IYC

9 CALL GYA (IYT,IYE,ICY)

10 CONTINUE

(F (I. EQ NY) GO TO 12

IYC=IXY(II)+ $\left.\left.\left\|^{\bullet}\right\| X Y \| 2\right)-\mid X Y \| 1\right\} \| W / N Y$

CALL GYA (IYT,IYB,IYC)

11 CONTINUE

12 RETURN

c

FORMAT $\left(0^{\circ} 0^{\circ} 10, \cdot\right.$ IN J530R. UPPER LIMIT INEAEASED BY $0.01 \%$

11

FORMAT $10^{\circ}$ A10. DECAOES EXCEEDS 25 IN J530A. REDUCE RANGE,RESU8MIT"

END

WLGLNO11

WLGLN012

WLGLN013

WLGLN014

WLGLN015

WLGLN016

WLGLN017

WLGLND18

WLGLNO19

WLGLNO20

WLGLNO21

WLGLN022

WLGLN023

WLGLN024

WLGLN025

WLGLNO26

WLGLN027

WLGLN02E

WLGLN029

WLGLNO30

WLGLNO31

WLGLNO32

WLGLNO33

WLGLN034

WLGLN035

WLGLNO36

WLGLNO37

WLGLN038

WLGLNO39

WLGLNO4O

WLGLNO41

WLGLNO42

WLGLNO43

WLGLNOA4

WLGLNO45

WLGLNOA6

WLGLNOAT

WLGLNDAB

WLGLNOA9

WLGLN050

WLGLNO51

WLGLN052

WLGLNO53

WLGLN054

WLGLNO55

WLGLN056

WLGLN057

WLGL V058

WLGLNDS9

WLGLNOSO

WLGLNOG1

WLGLNOS2

WLGLNOG3

WLGLNOS4

WLGLNO65

WLGLNO6E

WLGLNO67

WLGLNOG8

WLNLNOD1

WLNLNOO2

WLNLNOO3

NLNLNOO4

NLNLNO05

NLNLNOOS

WLNLNOOF

WLNLN008

WLNLNOO9 
CALL GYA (IYT,IYB,IXL)

WLNLN010

CALL GXA (IXL,IXR,IYB)

WLNLN011

NYS=MINO(NY,128)

DY=FLOAT(IYB- IYT)/NYS

IIXR=IXR- ITIC

NYS $=$ NYS -1

CALL GXA (IXL,IXR,IYT)

DO $1 \quad \mid=1$,NYS

IYS=IYB-1*DY

1 CALL DRV (IXR,IYS,IIXR,IYS)

DO $2 \quad l=1,19$

2 CALL GXA (IXL,IXR,ISC(I))

DO $3 \mathrm{I}=1,19$

CALL TSP $(80,1 S C(1), 5, F(1))$

RETURN

END

WLNLN012

WLNLN013

WLNLN014

WLNLN015

WLNLN016

WLNLN017

WLNLNO1B

WLNLNO19

WLNLN020

WLNLNO21

WLNLN022

WLNLN023

WLNLNO24

WLNLN025 


\section{APPENDIX B}

\section{EXAMPLE}

In this example, 14 data points from a 40 - item sam. ple are given. The lives of the 14 tailed units are chosen, within the limits of integer numbers of shots, equal to those derived from a sample of this size having the parameters

$$
\begin{aligned}
& \alpha=1000, \\
& \beta=0.8,
\end{aligned}
$$

and

$$
\gamma=400 .
$$

The maximum error is $0.32 \%$ for the fil weighted by the variance. The following pages show the input to the program, the printed outpul, and plots.

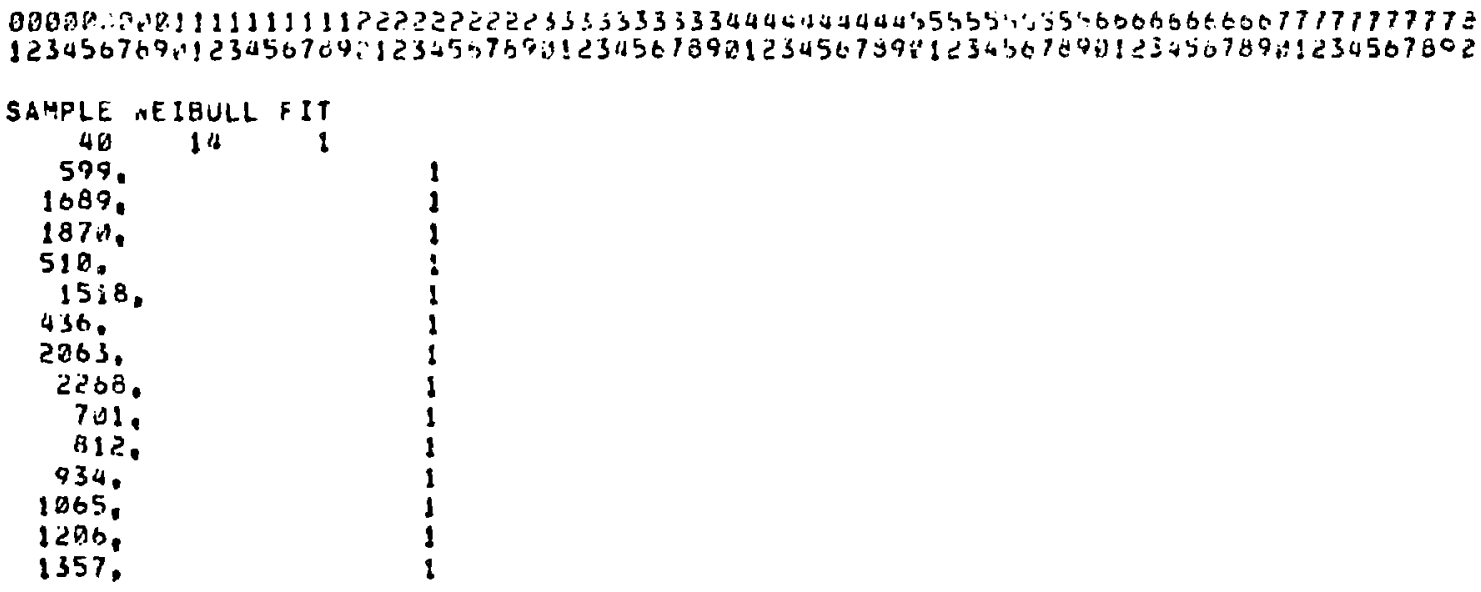

Program .nput

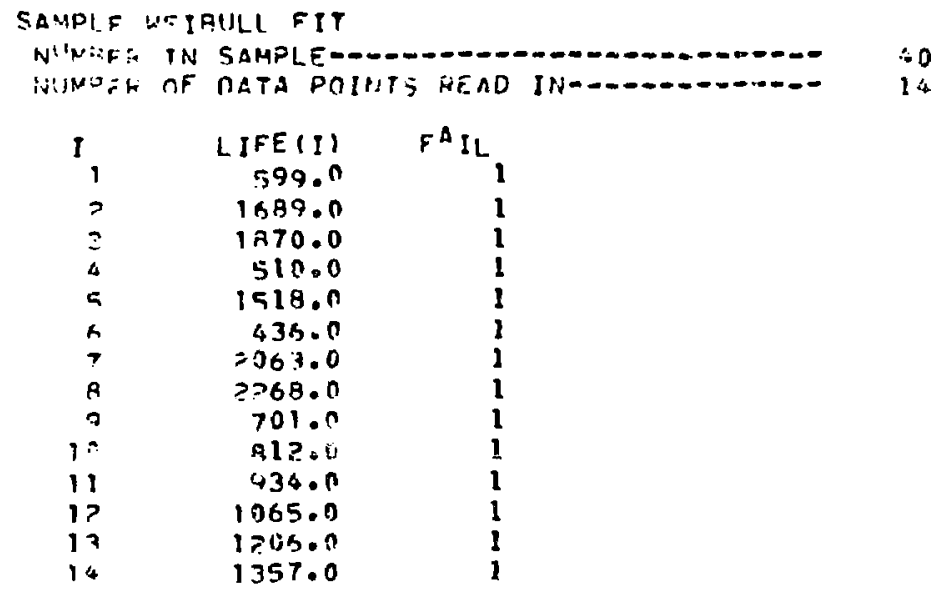

Program outpal page I 


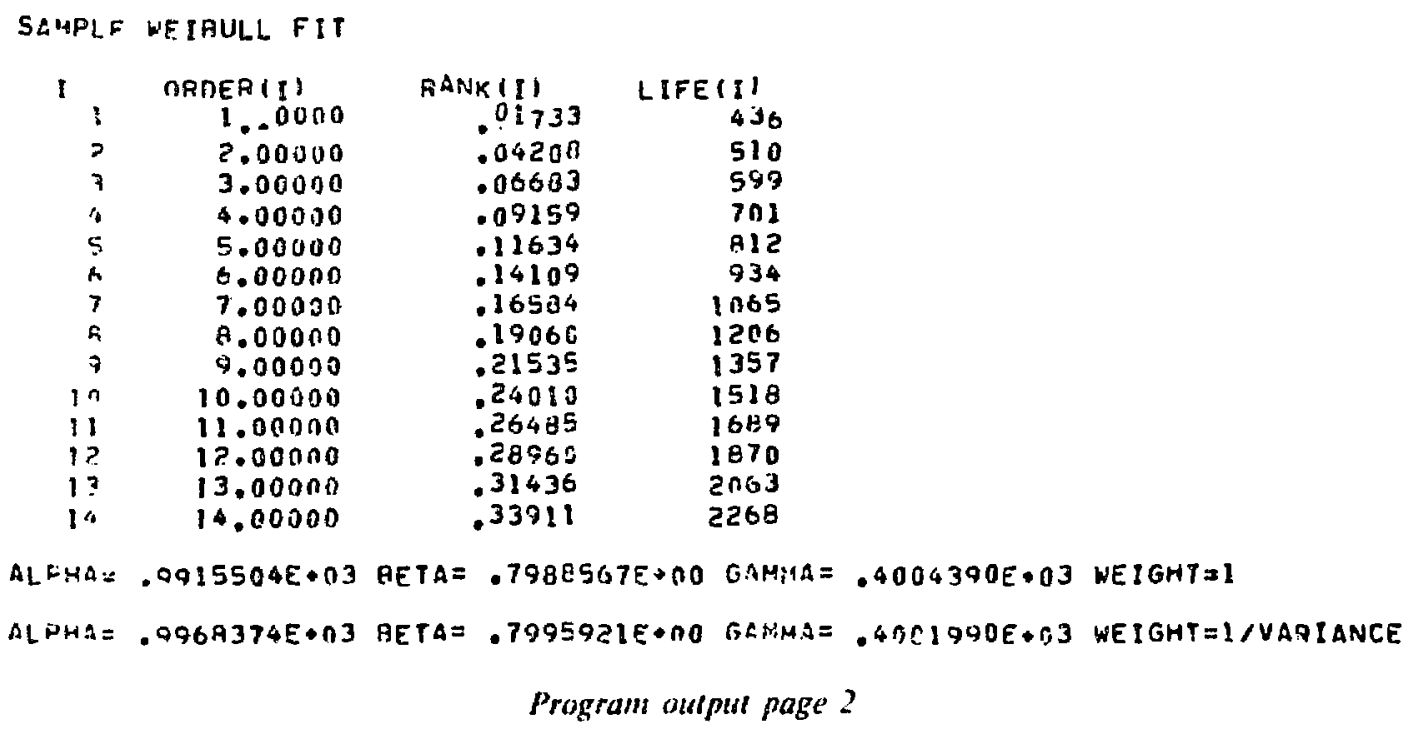




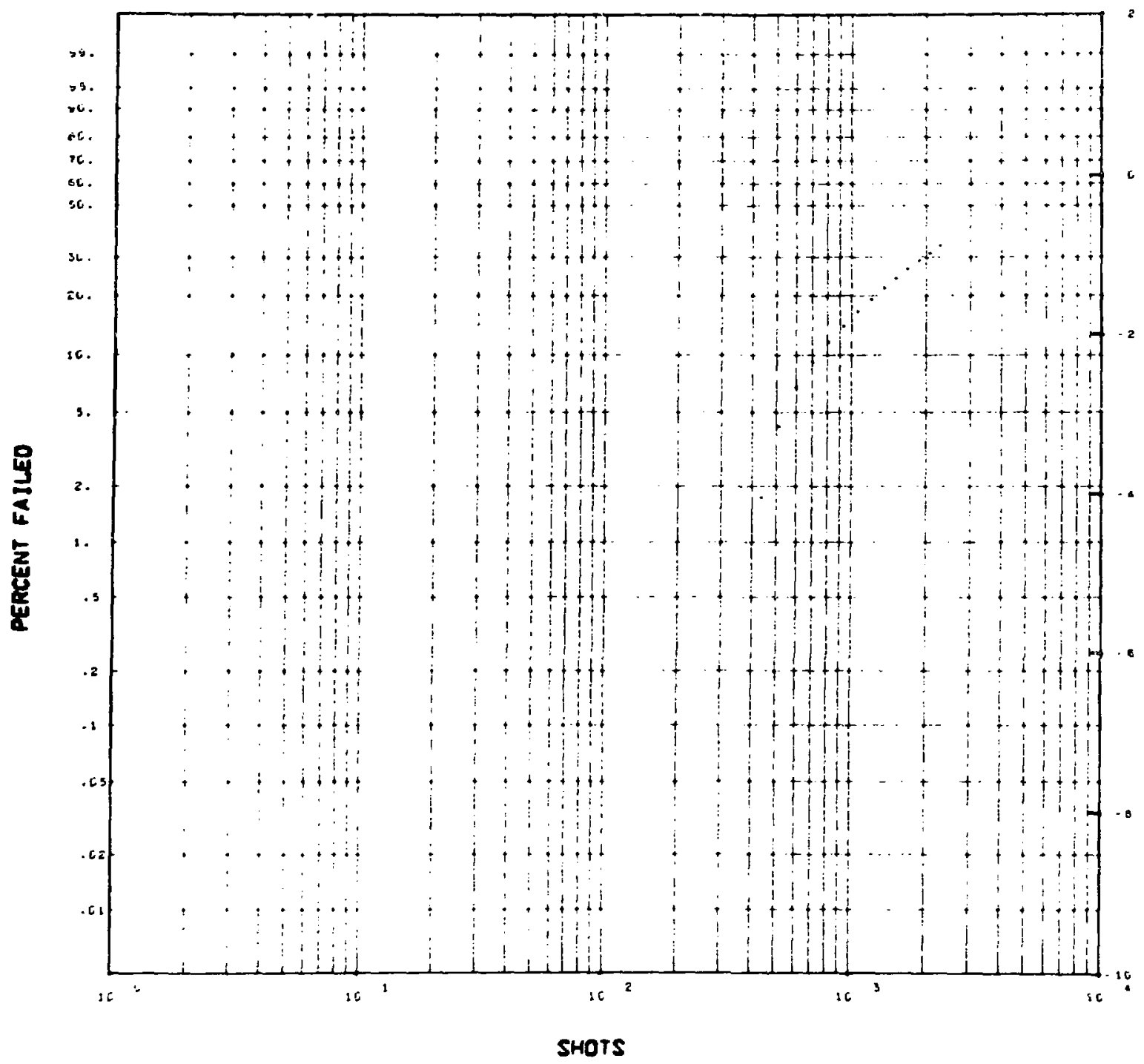

Film output frame 1 


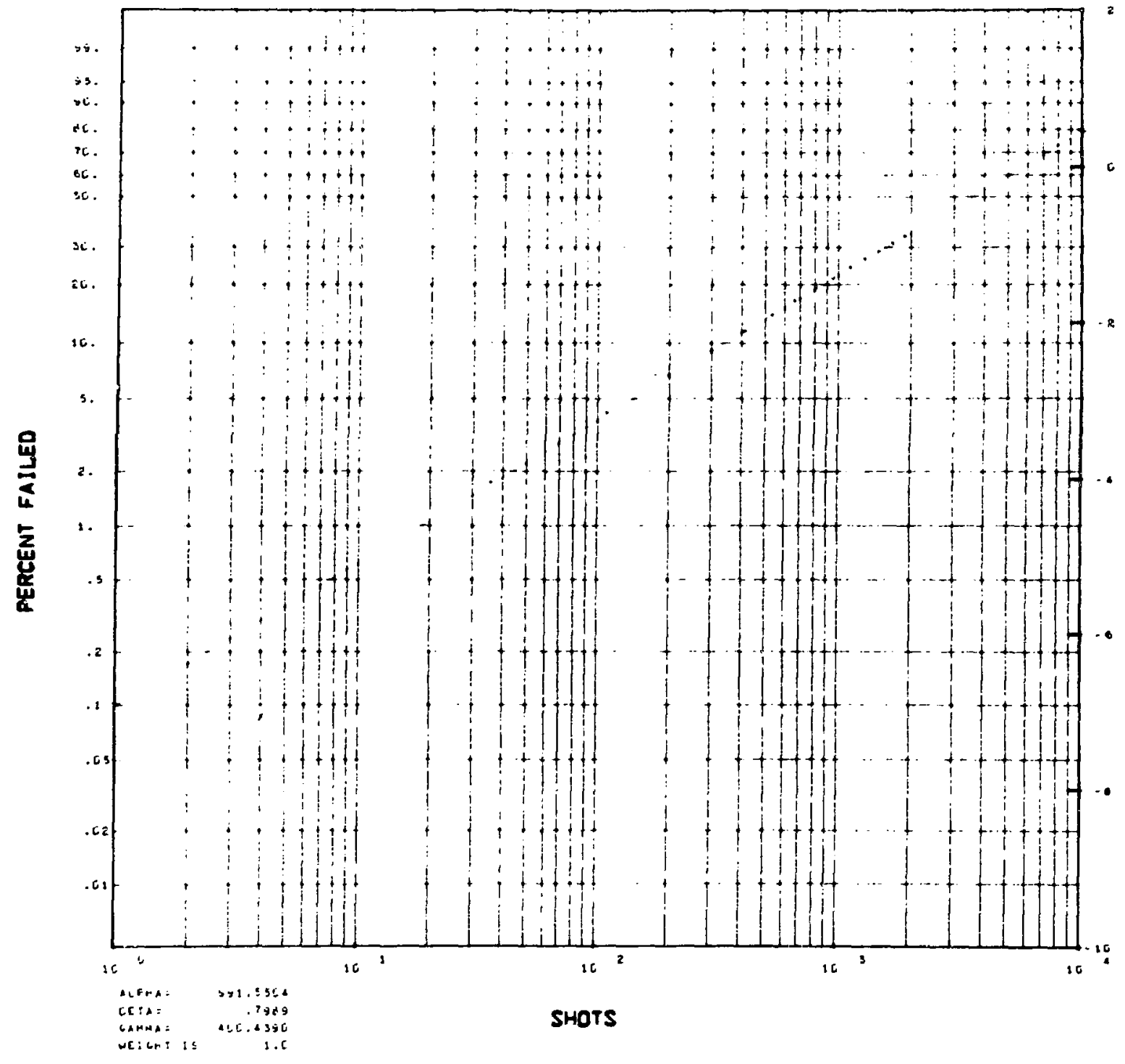

Fiim ouspus frame 2 


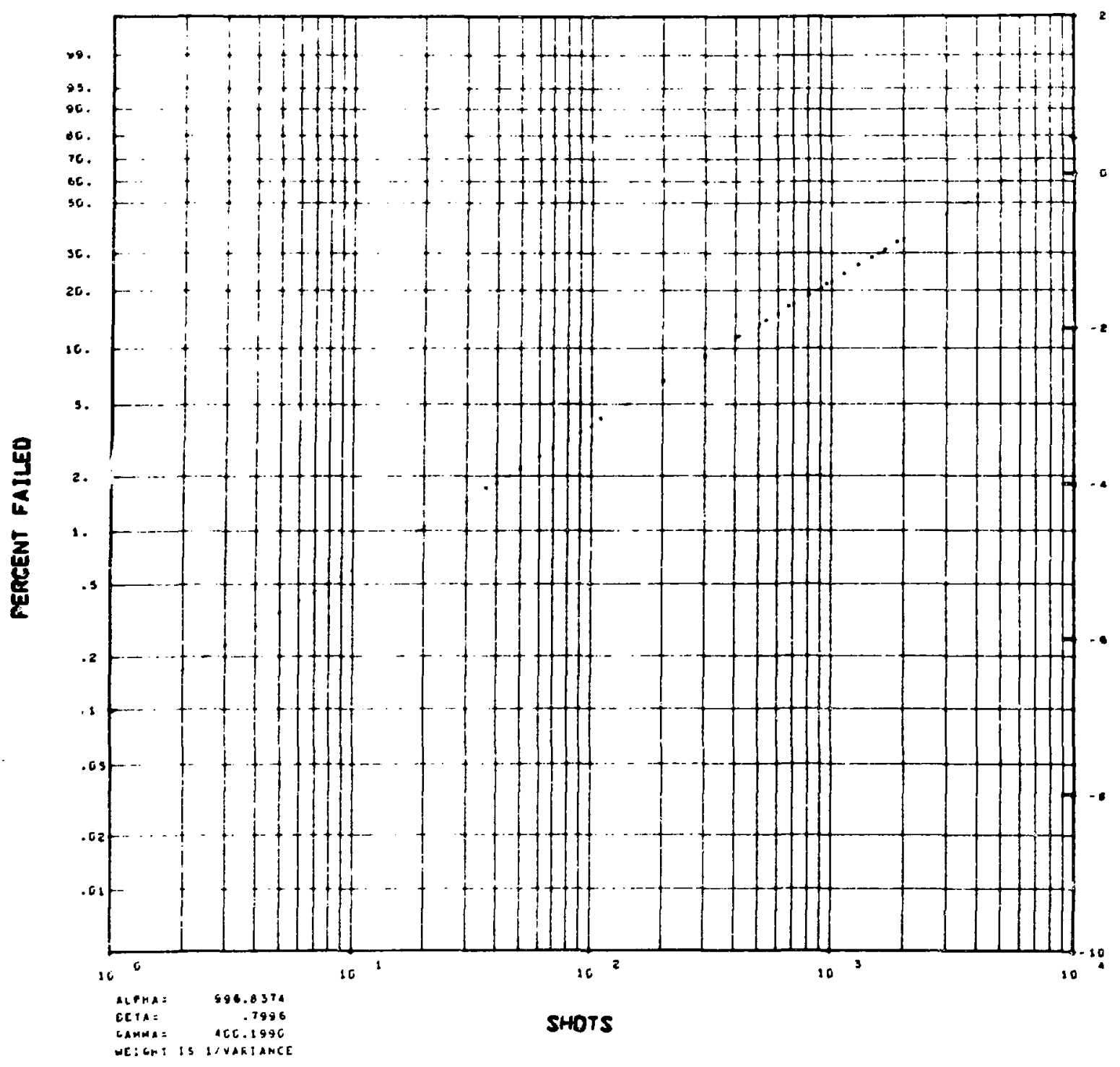

Film outpur frame 3 